\title{
ACTIVITÉ NYCTHÉMÉRALE ET UTILISATION DE LA SÉQUENCE RADIER-PROFOND PAR LES TRUITELLES D'UN AN (SALMO TRUTTA L.).
}

\author{
J.M. ROUSSEL et A. BARDONNET
}

INRA, Laboratoire d'Ecologie Aquatique, 65 rue de Saint-Brieuc, 35042 Rennes Cedex, France.

\begin{abstract}
RÉSUMÉ
Le comportement d'un groupe de dix truitelles d'un an (Salmo trutta L.) a été étudié au cours de deux cycles d'observation de 3 jours dans une séquence radierprofond reconstituée en milieu semi-naturel. Des changements de préférences d'habitat ont été mis en évidence à l'échelle du nycthémère et correspondent à des changements d'activité (repos, nage et alimentation). Lorsqu'elles sont en nage statique et en alimentation sur la dérive, les truitelles défendent leur poste et occupent le radier et le profond en pleine eau. Au repos, elles sont posées sur le fond du contre-courant et du profond, et elles n'exhibent pas de comportement agonistique. De plus, les truitelles ont montré un rythme d'activité diurne prononcé.
\end{abstract}

\section{DIEL ACTIVITY AND USE OF A RIFFLE/POOL UNIT BY ONE-YEAR-OLD BROWN TROUT (SALMO TRUTTA L.).}

\begin{abstract}
In the course of a 3-day study, the use of space of two groups of ten one-year-old brown trout (Salmo trutta L.) was investigated in the riffle/pool unit of an experimental stream. Diel changes in the location of fish were observed in relation with change in activity (resting, swimming and feeding). When free-swimming and feeding on drift, fish defended their position in the riffle and in the upper part of the water column of the pool. Resting fish were in the back eddy and on the bottom of the pool, and did not exhibit any agonistic behaviour. Fish were distinctly active during daytime.
\end{abstract}

\section{INTRODUCTION}

Comme la plupart des animaux, les salmonidés d'eau courante exhibent des préférences d'habitat qui s'expriment à différentes échelles spatiales, de la zone écologique (e.g. HUET, 1954) au micro-habitat (e.g. BOVEE, 1982). Si depuis une dizaine d'années, un nombre important d'études s'est attaché à définir les courbes de préférence pour tel ou tel paramètre physique de l'habitat, peu d'études ont porté sur la dimension temporelle de son utilisation. A l'échelle du nycthémère où s'exprime la succession quotidienne des activités du poisson, des changements de préférence d'habitat peuvent avoir lieu (CAMPBELL et NEUNER, 1985), entraînant des variations importantes dans les résultats obtenus lors de l'utilisation de modèles d'habitat (HARRIS et al., 1992). Cependant, si la relation habitat/activité nycthémérale a été décrite pendant la période hivernale (HEGGENES et al., 1993), elle ne l'a jamais été, à notre connaissance, aux autres saisons chez la truitelle d'un an. Notre hypothèse de travail est qu'il existe des différences d'utilisation de la séquence radier-profond au cours du nycthémère, et nous étudierons ici les changements de préférences d'habitat en fonction de l'activité réalisée. 


\section{MATÉRIEL ET MÉTHODES}

Les observations ont eu lieu du 27 mai au 7 juin 1994 dans un chenal expérimental à ciel ouvert de $10 \mathrm{~m}$ de long et $2,5 \mathrm{~m}$ de large, alimenté en circuit ouvert par le Lapitxuri, ruisseau de montagne affluent de la Nivelle (Pyrénées-Atlantiques). Une séquence radierprofond est reconstituée par aménagement du substrat de galets (Fig. 1). Pour un débit maintenu aux alentours de $0,12 \mathrm{~m}^{3} \cdot \mathrm{s}^{-1}$, on distingue quatre habitats aux caractéristiques mésologiques différentes:
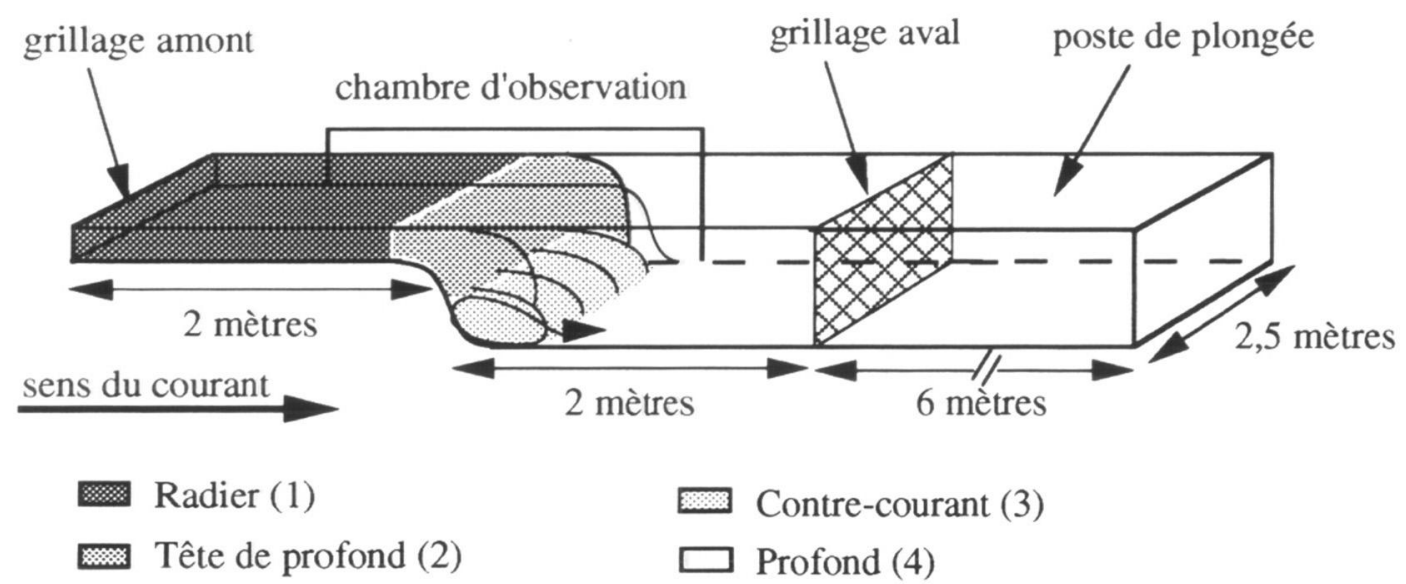

\section{Figure 1 : Représentation schématique du chenal d'étude.}

Figure 1 : Schematical drawing of the stream.

( 1 = riffle, 2 = head of the pool, 3 = back eddy, 4 = pool).

(1) le radier, où la vitesse de courant moyenne est de $30 \mathrm{~cm} \cdot \mathrm{s}^{-1}$ pour une profondeur de $16 \mathrm{~cm}$.

(2) la tête de profond, où le contraste vertical des vitesses est fort, de $30 \mathrm{~cm} . \mathrm{s}^{-1}$ en surface à pratiquement 0 près du contre-courant du profond.

(3) le contre-courant du profond, où la vitesse atteint $-7 \mathrm{~cm} \cdot \mathrm{s}^{-1}$ pour une profondeur de $50 \mathrm{~cm}$.

(4) le profond, où les vitesses sont en moyenne de $20 \mathrm{~cm} \cdot \mathrm{s}^{-1}$ en surface et $4 \mathrm{~cm} . \mathrm{s}^{-1}$ au fond, pour une profondeur de $55 \mathrm{~cm}$.

Une vitre latérale permet d'échantillonner le radier et l'amont du profond, un poste d'observation par plongée installé en aval de la séquence permettant d'échantillonner l'aval du profond (Fig.1). Deux lots de dix truitelles sauvages d'un an sont prélevés dans le Lapitxuri par pêche électrique et successivement étudiés dans le chenal. La longueur fourche moyenne pour le lot 1 est de $102 \mathrm{~mm}$ (de 95 à $112 \mathrm{~mm}$ ) et de $105 \mathrm{~mm}$ pour le lot 2 (de 99 à $115 \mathrm{~mm}$ ). Après trois jours de stabilisation, six séances d'observations directes, 3 de jour (à $10 \mathrm{~h}, 14 \mathrm{~h}, 18 \mathrm{~h}$ ) et 3 de nuit (à $23 \mathrm{~h}, 2 \mathrm{~h}$ et $5 \mathrm{~h}$ ) sont effectuées pendant trois jours. Elles durent en tout $40 \mathrm{mn}, 20 \mathrm{mn}$ depuis la chambre d'observation et $20 \mathrm{mn}$ en plongée. La température est relevée au début de chaque séance ; elle atteint $14^{\circ} \mathrm{C}$ en moyenne pendant l'étude, avec un minimum de $12.6^{\circ} \mathrm{C}$ le matin (relevé de $10 \mathrm{~h}$ ) et un maximum de $16.2^{\circ} \mathrm{C}$ l'après-midi (relevé de $18 \mathrm{~h}$ ). La position de chaque truitelle visible dans chacun des quatre habitats est relevée, en précisant la hauteur du poisson dans la colonne d'eau (sauf pour la tête de profond où la pente est trop forte pour une évaluation correcte étant donnée la mobilité des poissons) et l'activité du 
poisson : repos (poissons immobiles sur le fond), nage (poissons en nage statique dans la colonne d'eau ou poissons se déplaçant dans la séquence) et alimentation (poissons en nage statique capturant des proies sur la dérive ; aucun poisson s'alimentant sur le fond n'a été observé). Les observations de nuit sont faites en lumière blanche (50 W) avec une lampe étanche portable, permettant d'éclairer entièrement la séquence. L'observateur procède par flash de lumière de quelques secondes lui permettant de "photographier" rapidement la séquence, mais les données sur l'alimentation ne peuvent être récoltées.

Un dispositif de caméra vidéo infra-rouge placé dans la chambre d'observation permet d'enregistrer le nombre de passages et d'interactions agonistiques (chasses, morsures de caudale) en limite radier-profond sur un cycle de $24 \mathrm{~h}$. Pour le second lot, un enregistrement vidéo supplémentaire de $24 \mathrm{~h}$ en fin d'expérience en absence de l'observateur permet d'évaluer les éventuelles perturbations du groupe liées à la présence de l'observateur.

Les différences intra-lot (entre les 3 jours consécutifs d'observation de chaque lot) de fréquentation des 4 habitats, d'utilisation de la colonne d'eau et de proportion de truitelles en activité sont évaluées par le test de Kruskal-Wallis. Les différences inter-lots de répartition des truitelles dans la séquence sont évaluées pour chaque heure d'observation par le test de Mann-Whitney.

\section{RÉSULTATS}

Aucune différence intra-lot significative n'est détectée (test de Kruskal-Wallis, $p>0,05$ ). Pour clarifier la présentation des résultats, les données des 3 jours consécutifs d'observations directes sont donc regroupées heure par heure pour chacun des 2 lots.

\section{Nage et alimentation au cours du nycthémère}

Pour le lot 1, on note une forte proportion de truitelles en nage de jour (Fig. 2, $88 \%$ en moyenne pour les relevés diurnes), qui contraste avec les faibles proportions enregistrées de nuit ( $16 \%$ en moyenne). Les résultats pour le lot 2 concordent avec ce schéma, excepté pour le relevé de $10 \mathrm{~h}$ où la proportion de truitelles en nage est aussi faible $(14 \%)$ que pour les relevés nocturnes. Ainsi, les 2 lots étudiés sont-ils plutôt diurnes, avec une activité matinale $(10 \mathrm{~h})$ plus importante pour le lot 1 . Les résultats concernant l'alimentation sont assez bien corrélés avec l'activité de nage. Bien que l'alimentation nocturne n'ait pu être étudiée par observation directe, les enregistrements vidéo semblent indiquer que les poissons ne s'alimentent pas de nuit, au moins sur la dérive, la précision des images ne permettant pas d'être aussi catégorique pour une prise alimentaire benthique.

\section{Utilisation des quatre habitats}

De nuit, c'est l'ensemble contre-courant et profond qui accueille la presque totalité des truitelles (90\% en moyenne pour les 2 lots), et $55 \%$ des individus sont précisément dans le contre-courant (Fig. 3). Au contraire, la fréquentation du radier est plus forte (de 23 à $40 \%$ ) pendant les relevés de l'après-midi ( $14 \mathrm{~h}$ et $18 \mathrm{~h}$ pour le lot 2 , seulement $14 \mathrm{~h}$ pour le lot 1). La tête de profond, où les truitelles se positionnent face au flux sortant du radier, est presque exclusivement utilisée pendant les 2 relevés de l'après-midi.

D'après les histogrammes d'activité des truitelles (Fig. 2), on remarque que pendant les périodes d'activité $(10 \mathrm{~h}, 14 \mathrm{~h}$ et $18 \mathrm{~h}$ pour le lot $1 ; 14 \mathrm{~h}$ et $18 \mathrm{~h}$ pour le lot 2$)$, les individus se répartissent plus largement dans les 4 habitats disponibles, les fréquentations du radier et de la tête de profond étant alors maximales. Par contre, pendant les périodes de repos (relevés de nuit pour le lot 1 ; relevés de nuit et de $10 \mathrm{~h}$ pour le lot 2 ), les truitelles sont essentiellement dans le contre-courant et le profond. La différence inter-lots d'utilisation des 4 habitats n'est significative que pour le relevé de $10 \mathrm{~h}$ (test de MannWhitney, $p<0,05$ ), avec une affluence maximum de $67 \%$ en profond pour le lot 2 . 


\section{LOT 1}

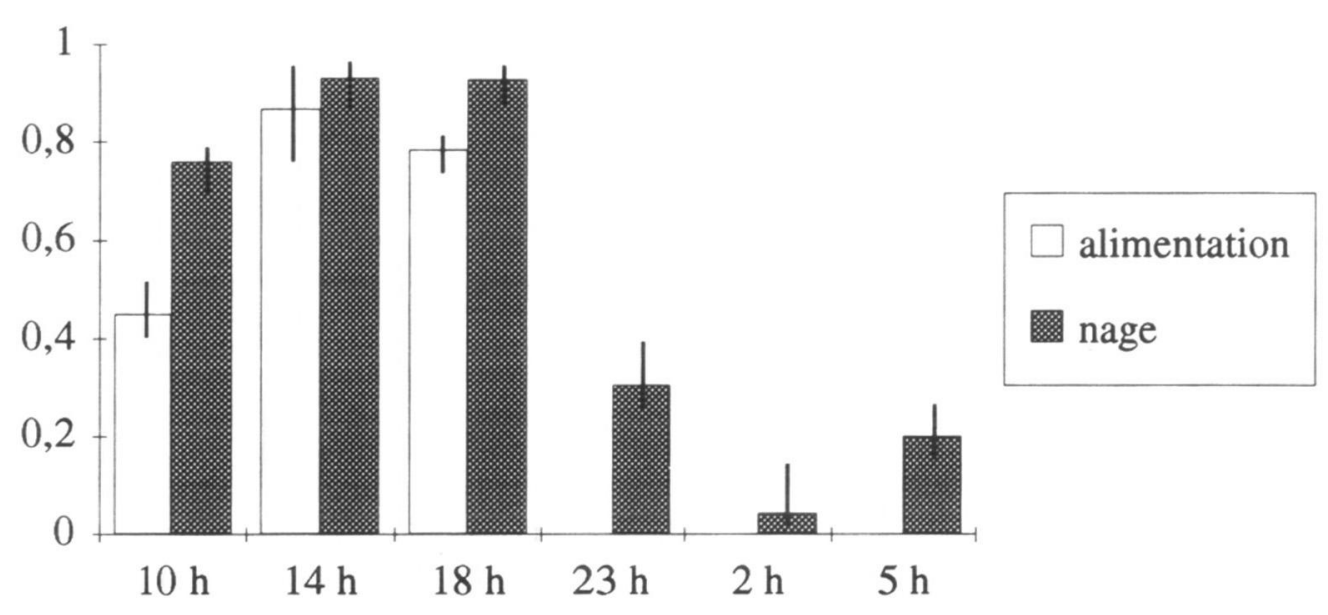

\section{LOT 2}

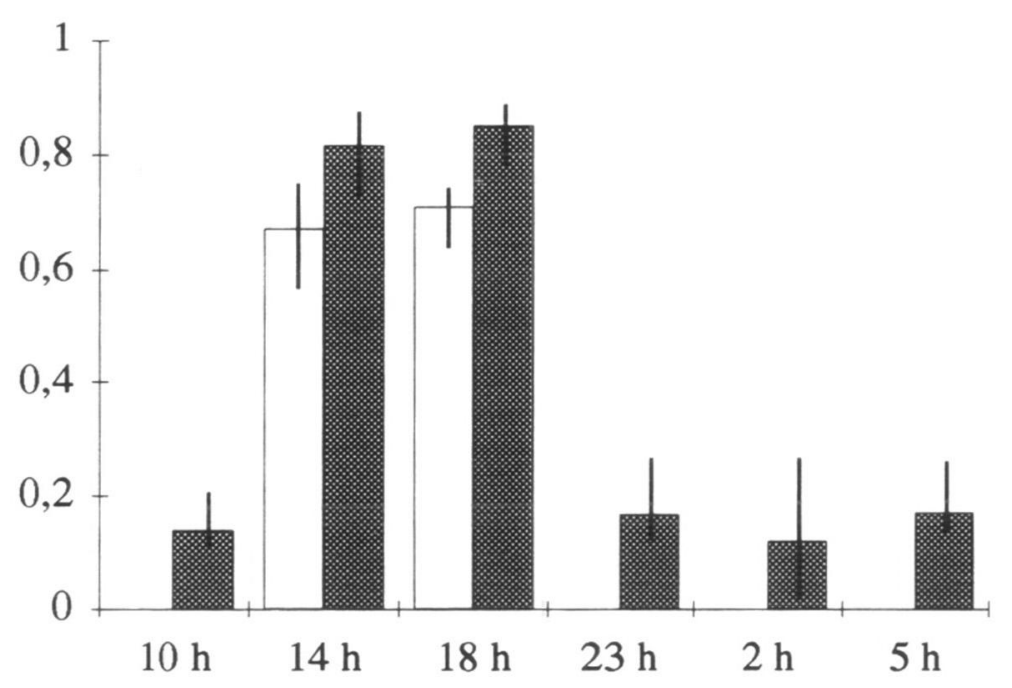

Figure 2 : Proportions de poissons en nage et en alimentation diurne sur la dérive aux heures d'observation directe. Les mini-maxi sont figurés par des barres verticales.

Figure 2 : Proportions of fish that swim free and drift feed during daylight at the time of direct observation surveys. Minimum and maximum are shown by vertical bars. 


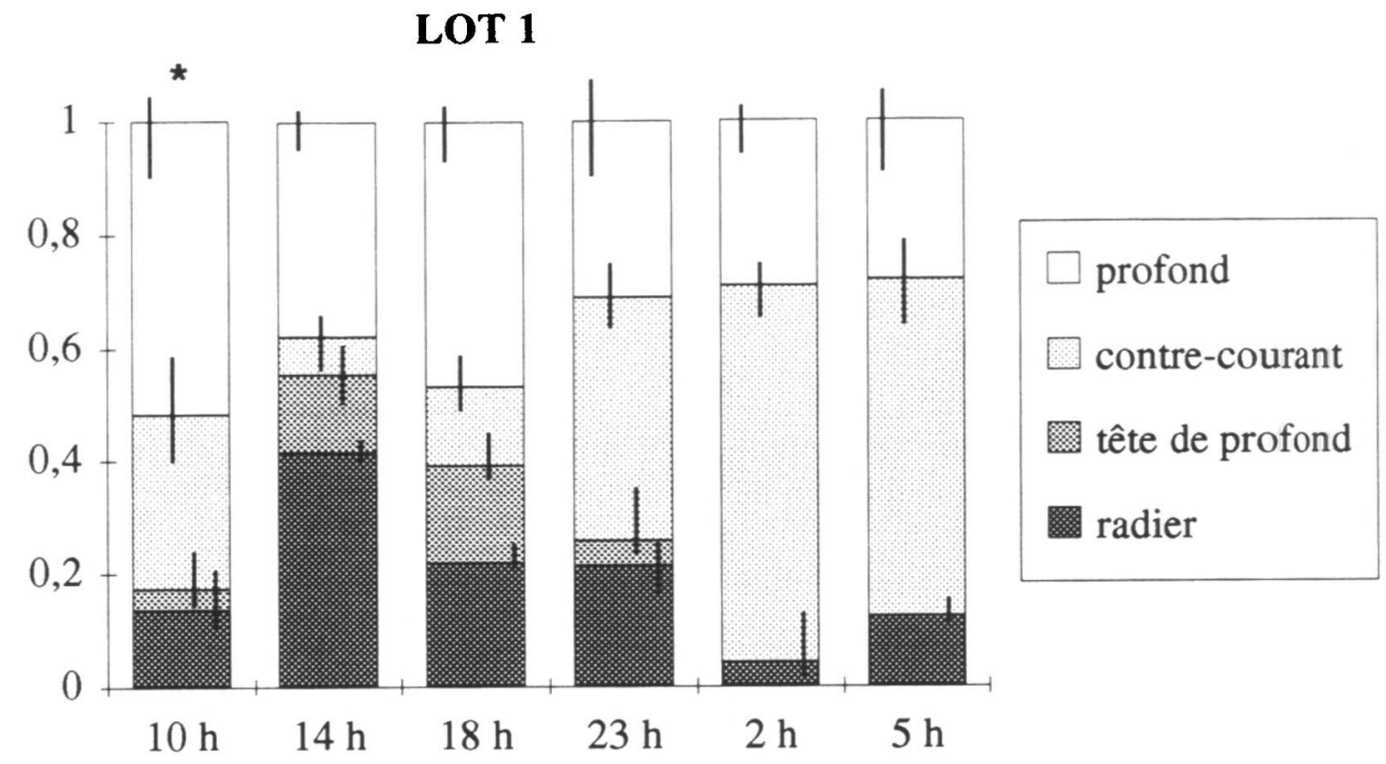

LOT 2

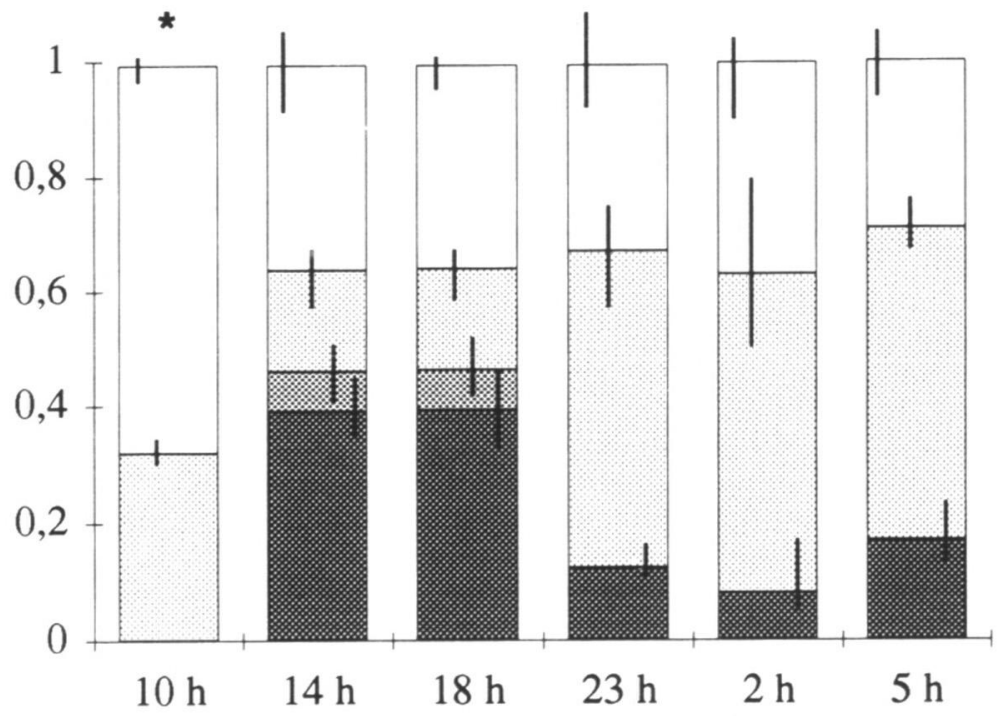

Figure 3 : Proportions de poissons présents dans les quatre habitats du chenal aux heures d'observation directe. Les mini-maxi sont figurés par des barres verticales. ${ }^{*}$ : différence significative entre les 2 lots, test de MannWhitney, $p<0,05)$.

Figure 3 : Proportions of fish present in the four habitats of the stream at the time of direct observation surveys. Minimum and maximum observed are shown by vertical bars. ${ }^{\star}$ : significant difference between the 2 lots, MannWhitney test, $p<0.05$ ). 


\section{Utilisation de la colonne d'eau}

Les truitelles présentes dans le radier sont toutes en nage statique à proximité du fond ( 2 à $5 \mathrm{~cm}$ ), dans la moitié inférieure de la colonne d'eau. Dans le profond et le contrecourant du profond, l'utilisation de la colonne d'eau (Fig. 4) s'ajuste bien aux courbes d'activité des truitelles (Fig. 2) : on constate qu'en période de repos (relevés de nuit pour le lot 1 ; relevés de nuit et de $10 \mathrm{~h}$ pour le lot 2 ), la majorité des truitelles ( 80 à $90 \%$ ) sont posées sur le fond, les autres étant dans la moitié inférieure de la colonne d'eau. Par contre, en période d'activité ( $10 \mathrm{~h}, 14 \mathrm{~h}$ et $18 \mathrm{~h}$ pour le lot $1 ; 14 \mathrm{~h}$ et $18 \mathrm{~h}$ pour le lot 2$)$, elles montent dans la colonne d'eau, tant en moitié inférieure (fréquentée par 25 à $50 \%$ des individus selon l'heure) qu'en moitié supérieure (de 25 à $50 \%$ également). On remarque à nouveau qu'à $10 \mathrm{~h}$, la différence inter-lots est significative (test de MannWhitney, $p<0,05$ ), les truitelles du lot 2 ayant un placement dans la colonne d'eau comparable à celui des relevés nocturnes.

\section{Enregistrement vidéo}

La confrontation des enregistrements 2 (présence de l'observateur) et 2 bis (absence de l'observateur) réalisés sur le lot 2 indique que la présence de l'observateur ne modifie pas le niveau d'activité du groupe le jour (Fig. 5). Par contre, il y a une perturbation du groupe pendant les observations de nuit, certainement en rapport avec l'utilisation de la lumière blanche : les petits pics enregistrés à $23 \mathrm{~h}$ et $2 \mathrm{~h}$ (moins de 10 passages par heure pour les lots 1 et 2) sont donc probablement des artefacts.

Les enregistrements confirment la très faible activité nocturne relevée pendant les observations directes (Fig. 2), en soulignant cependant l'existence d'une reprise d'activité dans l'heure précédant le lever du jour. II existe 3 périodes où les échanges entre radier et profond s'intensifient : le début de matinée, le début d'après-midi et la fin d'aprèsmidi/soirée. Après le pic d'activité qui suit le lever du jour, les truitelles semblent observer une "pause" plus ou moins importante (avec notamment un arrêt complet d'activité pour le lot 2), une seconde "pause" moins marquée intervenant vers $16 \mathrm{~h}$. La différence entre un lot 1 plus actif en début d'après-midi et un lot 2 inactif le matin et plus actif en fin de journée n'a pu être mise en relation avec les différences de température d'eau relevées entre les deux périodes d'expérimentation. On remarque que le nombre d'interactions agonistiques est assez bien corrélé avec le nombre de passages en limite radier-profond (sauf en début d'après-midi pour le lot 2).

\section{DISCUSSION}

Diverses études ont mis en évidence l'existence d'un rythme d'activité journalier chez la truite, mais celui-ci est variable, notamment en fonction de la saison (ERIKSSON, 1973 ; MÜLLER, 1978). Pour la période de fin de printemps et début d'été qui nous intéresse ici, les résultats de la littérature sont très contradictoires. La truite peut être essentiellement nocturne (CHASTON, 1968 ; CLAPP et al., 1990), présenter une activité de type bimodale au crépuscule et à l'aube (BACHMAN et al., 1979 ; ERIKSSON et ALANÄRÄ, 1992), ou encore être typiquement diurne (SWIFT, 1964). Dans les conditions de notre expérimentation, les truitelles d'un an ont montré un arrêt d'activité la nuit. Les enregistrements vidéo indiquent que cet arrêt est brutal et correspond exactement à la tombée de la nuit. Par ailleurs, le lever du jour induit la reprise des passages devant la caméra. II est donc probable que les niveaux d'activité nocturne quantifiés par observation directe, aient été exagérés par la technique d'observation. Si l'activité semble donc bien réduite à la photophase, elle n'est cependant pas constante tout au long de la journée. En observation directe, elle apparaît plus faible le matin, voire nulle pour le deuxième lot, et d'un niveau relativement comparable et important aux deux relevés de l'après-midi. Les enregistrements vidéo montrent 3 pics principaux (aube, début d'après-midi et fin de journée). Il est évidemment difficile de comparer les résultats issus de ces deux techniques, d'une part parce que les durées d'enregistrement sont différentes et d'autre part parce qu'on ne sait pas réellement ce que représente en terme d'activité le nombre de 


\section{LOT 1}

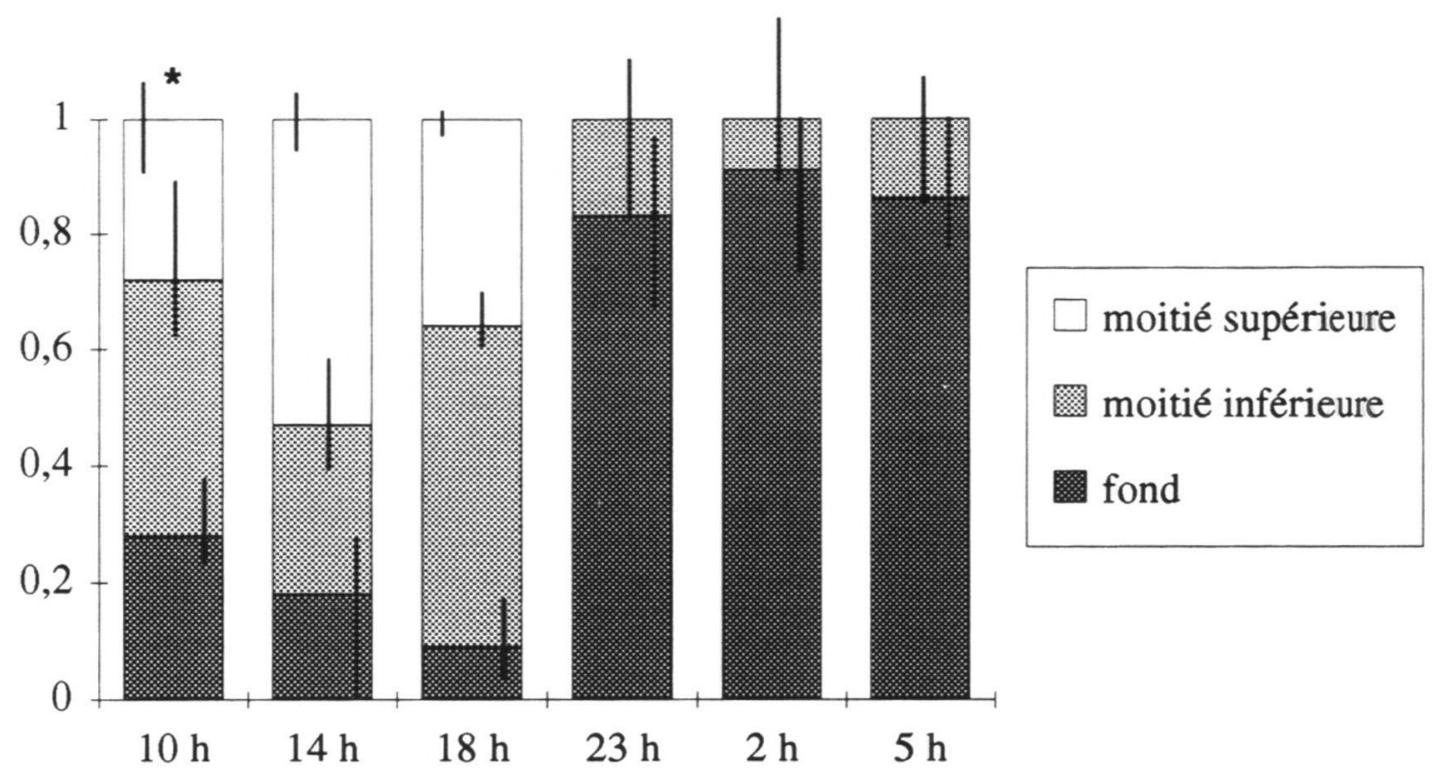

LOT 2

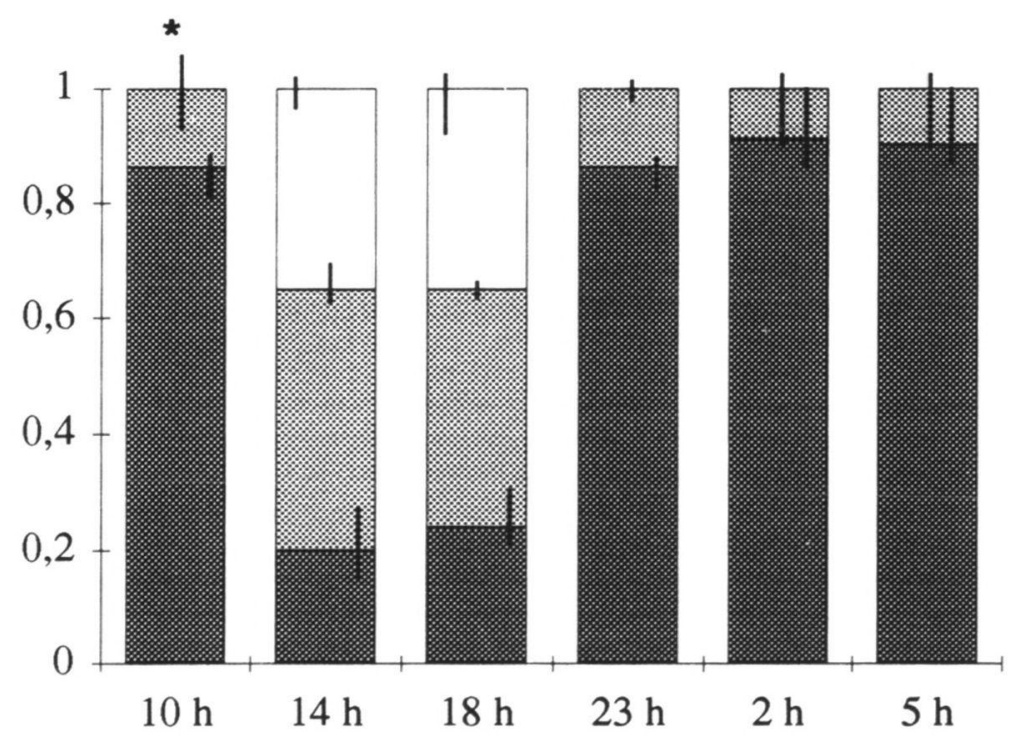

Figure 4 : Proportions de poissons posés sur le fond ou nageant dans les moitiés inférieure et supérieure de la colonne d'eau du profond et du contrecourant aux heures d'observation directe. Les mini-maxi sont figurés par des barres verticales. $\left(^{\star}\right.$ : différence significative entre les 2 lots, test de Mann-Whitney, $p<0,05)$.

Figure 4 : Proportions of fish in contact with the substratum or swimming in the lower or upper part of the water column of the pool and in the back eddy at the time of direct observation surveys. Minimum and maximum observed are shown by vertical bars. ( ${ }^{*}$ : significant difference between the 2 lots, Mann-Whitney test, $p<0.05)$. 

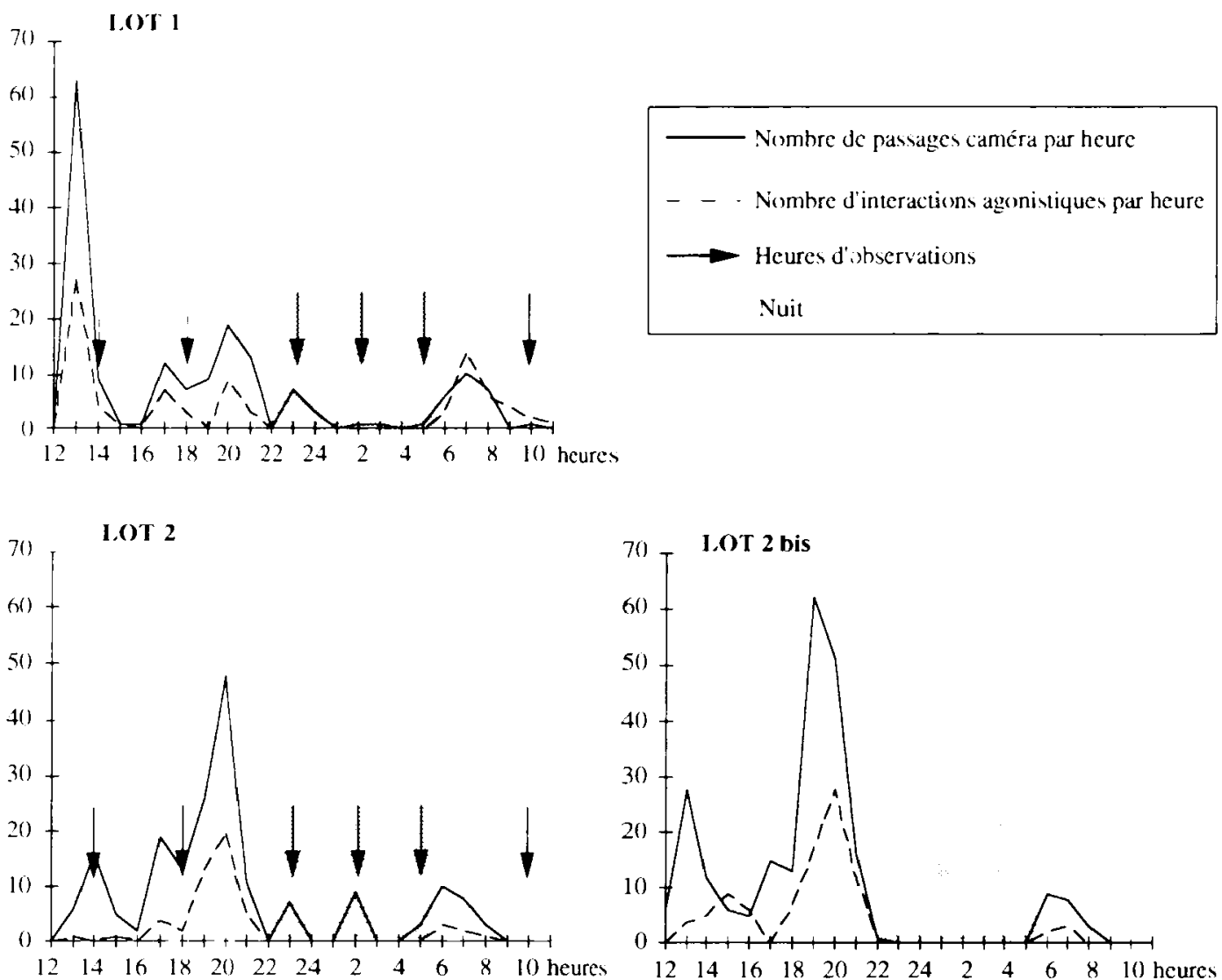

Figure 5 : Nombres de passages devant la caméra et d'interactions agonistiques exprimés en unités par heure pendant $24 \mathrm{~h}$ pour le lot 1 et pour le lot 2 . Le lot 2 bis est un enregistrement de contrôle en absence de toute observation directe.

Figure 5 : Numbers of crossing through the camera field and of agonistic behaviour expressed in units per hour during 24 hours for fish of lot 1 and lot 2 . Lot 2 bis is a video-sample made without any direct observation survey to test disturbance caused by the observer.

passages devant la caméra. Malgré tout, les différences dans les conclusions issues de l'une ou l'autre des techniques de prises de données n'apparaissent pas contradictoires. II est troublant de constater à quel point les pics correspondants aux nombres de passages devant la caméra interviennent aux mêmes heures pour les deux lots. II existe peut-être ici, en plus de la période de 24 heures liée au lever du jour et à la tombée de la nuit, des rythmes infra-circadiens du niveau d'activité du groupe, liés par exemple à la physiologie des poissons (phénomène de satiété). Cependant, il peut aussi s'agir d'une réponse à des phénomènes circadiens tels que, par exemple, les maxima d'éclairement et de température.

Ces variations du niveau d'activité des truitelles sont associées à un net changement de leur comportement et de leurs préférences d'habitat au cours du nycthémère. Pendant les périodes d'alimentation sur la dérive, il y a une plus large répartition des truitelles dans 
l'habitat disponible et une augmentation de l'agressivité. Ces manifestations sont probablement le résultat du comportement de territorialité, qui a été abondamment décrit chez les salmonidés (HÉLAND, 1977 ; PUCKETT et DILL, 1985 ; GRANT et KRAMER, 1990). Pendant les périodes de repos, l'agressivité disparaît et les truitelles se regroupent dans le contre-courant et le profond. On peut faire le parallèle avec les résultats de CUNJAK et POWER (1986) qui ont montré un changement de préférences d'habitat entre l'hiver et l'été comparable à celui observé ici à l'échelle journalière. L'agressivité intra-spécifique qui implique une dépense d'énergie ne serait observée qu'en période de forte activité, à savoir au cours des saisons dites à forte croissance et seulement pendant les phases d'alimentation. Pendant la baisse hivernale d'activité (HEGGENES et al., 1993), tout comme pendant les phases de repos aux autres saisons, les truites adopteraient une stratégie non consommatrice d'énergie, à savoir une meilleure tolérance de leurs congénères.

Enfin, le principal habitat de repos nocturne (contre-courant du profond) représente un volume très réduit : $60 \%$ des poissons se retrouvent en effet dans seulement $6 \%$ de la surface totale. Ainsi, à densité égale, les surfaces nécessaires à la réalisation de l'activité de repos sont comparativement plus faibles que celles nécessaires à l'activité d'alimentation sur la dérive. Si l'évaluation de la capacité d'accueil des rivières doit considérer les habitats nécessaires à la réalisation des différentes activités du poisson (alimentation, repos), elle doit aussi considérer les possibles changements comportementaux associés à ces activités.

\section{REMERCIEMENTS}

Nous remercions M. HÉLAND et E. BEALL (INRA, St Pée-sur-Nivelle) pour leurs conseils au cours de l'expérimentation, ainsi que S. GLISE, M. PARADE et J.C. VIGNES (INRA, St Pée-sur-Nivelle) pour leur assistance technique.

\section{BIBLIOGRAPHIE}

BACHMAN R.A., REYNOLDS W.W., CASTERLIN M.E., 1979. Diel locomotor activity patterns of wild brown trout (Salmo trutta) in an electronic shuttlebox. Hydrobiologia, $66,45-47$.

BOVEE K.D., 1982. A guide to stream analysis using the Instream Flow Incremental Methodology. Instream Flow Information Paper $n^{\circ} 12$, US Fish and Wildlife Service FWS/OBS 82/26, $248 \mathrm{p}$.

CAMPBELL R.F., NEUNER J.D., 1985. Seasonal and diurnal shifts in habitat utilized by resident rainbow trout (Salmo gairdneri) observed in western Washington cascade mountain streams. In OLSON and WHITE, Proceedings of symposium on small hydropower and fisheries, 38-49, American Fisheries Society, Denver.

CHASTON I., 1968. Influence of light on activity of brown trout (Salmo trutta). Can. J. Fish. Res. Board Can., 25, 1285-1289.

CLAPP D.F., CLARK R.D., DIANA J.S., 1990. Range, activity and habitat of large, freeranging brown trout in Michigan stream. Trans. Am. Fish. Soc., 119, 1022-1034.

CUNJAK R.A., POWER G., 1986. Winter habitat utilization by stream resident brook trout (Salvelinus fontinalis) and brown trout (Salmo trutta). Can. J. Fish. Aquat. Sci., 43, 1970-1981.

ERIKSSON L.O., 1973. Spring inversion of the diel rhythm of locomotor activity in young sea-going brown trout, Salmo trutta trutta $\mathrm{L}$. and atlantic salmon, Salmo salar $\mathrm{L}$. Aquilo Ser. Zool., 14, 68-79. 
ERIKSSON L.O., ALANÄRÄ A., 1992. Timing of feeding behavior in salmonids. In THORPE J.E., HUNTINGFORD F.A., The importance of feeding behavior for the efficient culture of salmonid fishes, 41-48, The World Aquaculture Society Publ., Baton Rouge.

GRANT J.W.A., KRAMER D.L., 1990. Territory size as a predictor of the upper limit to population density of juvenile salmonids in streams. Can. J. Fish. Aquat. Sci., 47, 1724-1737.

HARRIS D.D., HUBERT W.A., WESCHE T.A., 1992. Habitat use by young-of-the-year brown trout and effects on Weight Usable Area. Rivers, 3, 99-105.

HEGGENES J., KROG O.M., LINDAS O.R., DOKK J.G., BREMNES T., 1993. Homeostatic behavioural responses in a changing environment : brown trout (Salmo trutta L.) become nocturnal during winter. J. Anim. Ecol., 62, 295-308.

HÉLAND M., 1977. Recherches sur l'ontogenèse du comportement territorial chez l'alevin de truite commune. Thèse de doctorat de 3 ème cycle, Université de Rennes, Rennes, $239 \mathrm{p}$.

HUET M., 1954. Biologie, profils en long et en travers des eaux courantes. Revue Suisse d'Hydrobiologie, 11, 332-351.

MÜLLER K., 1978. Locomotor activity of fish and environmental oscillations. In THORPE J.E., Rhythmic activity of fishes, 1-19, Academic press, London.

PUCKETT K.J., DILL L.M., 1985. The energetics of territoriality in juvenile coho salmon (Oncorhynchus kisutch). Behaviour, 92, 97-111.

SWIFT D.R., 1964. Activity cycles in the brown trout (Salmo trutta L.). 2 - Fish artificially fed. J. Fish. Res. Bd Can., 21, 133-138. 